\title{
APORTACIONES A LA ZOOGEOGRAFÍA DEL HAYEDO DEL MOCAYO
}

\author{
Luis Alberto LONGARES ALADRÉN \\ Departamento de Geografía y Ordenación del Territorio \\ Universidad de Zaragoza \\ Pedro LOZANO VALENCIA \\ Departamento de Geografía \\ Universidad del País Vasco \\ José Manuel RUBIO RECIO \\ Departamento de Geografía \\ Universidad de Sevilla
}

\begin{abstract}
Resumen: En éste trabajo, se plantea el estudio zoogeográfico del hayedo del Moncayo (Aragón, España), en relación con la situación límite de distribución de dicha formación vegetal. Su comparación con la fauna detectada en diferentes hayedos, permite detectar presencias y ausencias faunísticas significativas, las cuales, indican la situación ecológica y el verdadero carácter corológico de esta masa boscosa sometida a estudio.
\end{abstract}

Palabras clave: Zoogeografía, corología, fauna, hayedo.

Abstract: The paper explains a zoogeographic study in the Moncayo beechwood (Aragón, Spain) in relation with the border tree situation of this vegetal group. Significant fauna presences and absences were detected comparing with other beechwoods which indicate the ecological position and the real corological value of this site.

Key words: Zoogeography, corology, fauna, beechwood.

\section{INTRODUCCIÓN Y OBJETIVOS}

Durante el transcurso de las Jornadas de Biogeografía, celebradas del 7 al 13 de junio de 1997 en la localidad de San Martín del Moncayo, provincia de Zaragoza, el 
grupo dedicado al ámbito de la Zoogeografía se planteó como objetivo desarrollar técnicas de muestreo y censo de dicha disciplina.

Los resultados obtenidos durante estos días, completados con estudios realizados en la zona, dieron lugar al presente trabajo al plantearse la duda acerca de la posible influencia, por su ubicación relicta o de límite de distribución de la formación boscosa de Fagus sylvatica, en la presencia o ausencia de determinada avifauna ligada a éste biotopo.

\section{DESCRIPCION DEL AREA DE ESTUDIO}

El Macizo del Moncayo, situado en el sector zaragozano de la Cordillera Ibérica, constituye un enclave donde están presentes una parte importante de los ecosistemas de nuestro país. Su amplitud altitudinal y su situación geográfica entre condiciones eurosiberianas y mediterráneas, permiten la coexistencia de especies con orígenes biogeográficos distintos y exigencias ecológicas diversas, que hace que algunos de sus ecosistemas estén considerados, por algunos autores, como áreas de distribución relictual, con enclaves atlánticos o eurosiberianos inmersos en un mundo mediterráneo y submediterráneo, mientras que otros hablan de situaciones de continuidad con enclaves presentes en el Sistema Ibérico.

Las características bioclimáticas que presenta el área de estudio, en su vertiente noroeste (elevado gradiente altitudinal, que provoca un aumento de las precipitaciones con la altitud y una mayor exposición a los vientos húmedos de poniente), permiten la presencia de un enclave florístico de características eurosiberianas como el hayedo del Moncayo (Mapa1), en el interior de un ambiente de dominio mediterráneo continentalizado, lo cual ha llevado a diversos autores a considerarlo como relicto (GÓMEZ, 1997; MONTSERRAT, 1984), o simplemente como el límite meridional de los situados en las vertientes más húmedas del Sistema Tbérico en su sector noroccidental (BALCELLS, 1993).

El área de estudio elegida, se sitúa en la ladera noroeste del Moncayo, entre 1.300 y $1.600 \mathrm{~m}$. de altitud. Se trata de un bosque de hayas, incluido dentro de la asociación Ilici aquifolii-Fagetum, caracterizado como un hayedo de tipo oligotrofo, en relación con sustratos metamórficos, con una importante influencia mediterránea en su cortejo florístico, pero relacionado estrechamente con los hayedos cantábricos (GÓMEZ, 1997); muestra de ello es la presencia de bosquetes de abedules ligados a los pequeñas torrenteras que circulan por el interior del hayedo (FERRERAS, 1989). 


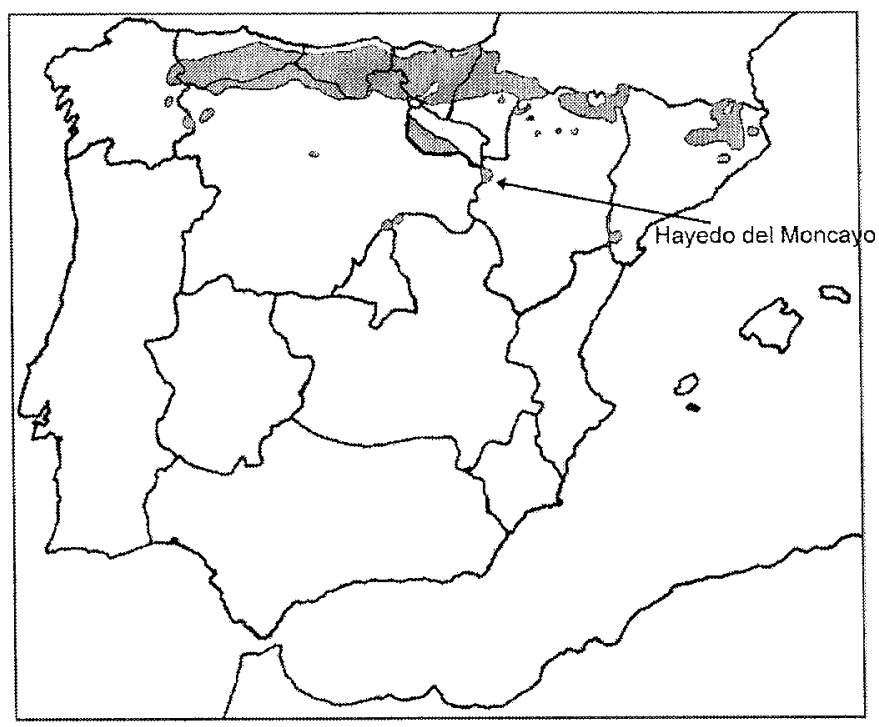

Mapa 1. Localización los principales hayedos en la Península Ibérica y ubicación del área de estudio (Elaboración propia).

Se ubica en la zona conocida como el barranco de Castilla, ocupando una banda reducida debido a una explotación antigua de su parte más alta. Se trata de un bosque aclarado de forma sucesiva, que según GÓMEZ (1960) históricamente tendría una distribución similar a la actual, aunque ocuparía una mayor extensión. Este aprovechamiento ha provocado la existencia de un bosque joven, con un pequeño enclave primitivo en su zona más inaccesible (Barranco de Castilla), que presenta una importante lixiviación de su suelo y por lo tanto acidificación por la lluvia en época de poca evaporación, situación que no se daría si se tratara de un hayedo maduro, el cual frenaría el lavado del ; esto se deduce por la presencia de brezos y otras plantas acidófilas en zonas de mayor encharcamiento, donde las hayas no arraigan con facilidad al encharcarse sus raíces, colonizando este espacio avellanos, sauces y abedules (MONTESERRAT, 1984).

\section{METODOLOGÍA}

La duración de las Jornadas no permite, de ningún modo, hacer un análisis exhaustivo de la fauna, pero si aproximarnos a la componente zoogeográfica de este espacio. 
Tabla 1.- Aviafauna citada en hayedos eurosiberianos.

\begin{tabular}{|c|c|c|c|c|c|c|c|c|}
\hline ESPECIES & & $\begin{array}{l}\text { Avifauna } \\
\text { Hayedos } \\
\text { (Gómez) }\end{array}$ & $\begin{array}{c}\text { Avifauna } \\
\text { Hayedos } \\
\text { (Pedrocchi) }\end{array}$ & $\begin{array}{c}\text { Avifauna } \\
\text { Hayedos } \\
\text { (Balcells) }\end{array}$ & $\begin{array}{c}\text { Nidificante } \\
\text { Hayedos } \\
\text { (Purroy) }\end{array}$ & $\begin{array}{c}\text { Avifauna } \\
\text { Hayedos } \\
\text { (Costa) }\end{array}$ & $\begin{array}{c}\text { Avifauna } \\
\text { Hayedos } \\
\text { (Costa) }\end{array}$ & $\begin{array}{c}\text { Avifauna } \\
\text { Hayedos } \\
\text { (Costa) }\end{array}$ \\
\hline Nombre común & Nombre científico & P. Ibérica & \begin{tabular}{|c|} 
Pirineo \\
Occidental
\end{tabular} & $\begin{array}{c}\text { Parque Nac. } \\
\text { Ordesa }\end{array}$ & Pirineo & $\begin{array}{l}\text { Valdeon } \\
\text { (León) }\end{array}$ & $\begin{array}{l}\text { Sajambre } \\
\text { (León) }\end{array}$ & $\begin{array}{l}\text { Buron } \\
\text { (León) }\end{array}$ \\
\hline Azor & Accipiter gentilis & Presente & Presente & & Presente & & & Presente \\
\hline Gavilán & Accipiter nisus & Presente & Presente & & Presente & Presente & & \\
\hline Mito & Aegithalos caudatus & - & - & & Presente & Presente & Presente & Presente \\
\hline Bisbita arbóteo & Anthus trivinlis & Presente & - & & & & & \\
\hline Búlho chico & Asio otus & Presente & - & & & & & \\
\hline Aguila ratonera & Buteo buteo & Presente & Presente & & Presente & Presente & Presente & Presente \\
\hline Chotacabras gris & Caprinulgus ewropaeus & - & - & & + & & & \\
\hline Jilguero & Carduelis carduelis & & & & & Presente. & & \\
\hline Lúgano & Carduelis spinus & - & - & Presente & - & Presente & Presente & \\
\hline Agateador común & Certhia brachydactya & Presente & Ptesente & Presente & Frecuente & & Presente & \\
\hline Agateador norteño & Certhis familiaris & Presente & Presente & Presente & Frecuente & Presente & Presente & Presente \\
\hline Aguila culcbrera & Circaetus gallicus & - & Presente & & - & & & \\
\hline Picogordo & Coccothraustes coccothraustes & & & & & Presente & Presente & Presente \\
\hline Paloma zurita & Columba oenas & - & Presente & & - & & & \\
\hline Paloma torcaz & Columba palumbus & - & Presente & & Presente & Presente & Presente & Presente \\
\hline Cuervo & Convus corax & - & - & & Presente & Presente & Presente & \\
\hline Corneja & Con'us corone & - & - & & Presente & Presente & Presente & Presente \\
\hline Cuco & Cuculus canorus & - & Presente & & Presente & Presente & Presente & Presente \\
\hline Pico dorsiblanco & Dendrocopos leucotos & - & Presente & Presente & Presente & & & \\
\hline Pico picapinos & Dendrocopos major & - & Presente & & Presente & Presente & Presente & Presente \\
\hline Pito negro & Dryocopus martius & - & Presente & Presente & Presente & Presente & Presente & Presente \\
\hline Escribano montesino & Emberiza cia & - & - & & $\cdot$ & Presente & Presente & \\
\hline Escribano cerillo & Emberiza citrinella & - & - & Presente & - & & & \\
\hline Petirrojo & Erithacus rubecula & Presente & Presente & & Abundante & Presente & Presente & Presente \\
\hline Cernícalo cornún & Falco tinunnunculus & - & - & & - & & & \\
\hline Papamoscas cerrojillo & Ficedula hypoleuca & Presente & - & & Presente & Presente & Presente & \\
\hline Pinzón & Fringilla coelebs & - & Presente & & Abundante & Presente & Presente & Presente \\
\hline Arrendajo & Garrulus glandarius & Presente & Presente & & Presente & Presente & Presente & Presente \\
\hline Aguila calzada & Hieratus pennatus & Presente & - & & - & & & \\
\hline Alcaudón dorsirrojo & Lanius collurio & Presente & - & Presente & & & & \\
\hline Milanoreal & Milvus milvus & - & Presente & & - & & & \\
\hline Papamoscas gris & Muscicapa striata & - & Presente & & - & & & \\
\hline Carbonero garrapinos & Parus ater & - & Presente & & Abundante & Presente & Presente & Presente \\
\hline Herrerillo & Parus caeruleus & - & Presente & & \begin{tabular}{|l|} 
Freculente \\
\end{tabular} & Presente & Presente & Presente \\
\hline Henerillo capuchino & Parus cristatus & Presente & Presente & & Escasa & Presente & Presente & Presente \\
\hline Carbonero conún & Parus major & - & Presente & & Frecuente & Presente & Presente & Presente \\
\hline Carbonero palustre & Parus palustris & Presente & Presente & Presente & Frecuente & Presente & Presente & Presente \\
\hline HalcÛn abejero & Penis apivorus & Presente & Presente & & - & & & \\
\hline Mosquitero papialbo & Phylloscopus bonelli & & & & & & & Presente \\
\hline Moquitero común & Phylloscopus collybita & - & Presente & & Presente & Presente & Presente & Presente \\
\hline \begin{tabular}{|l|} 
Pito real \\
\end{tabular} & Picus viridis & - & Presente & & Presente & Plesente & & \\
\hline Acentor & Prwnella modularis & - & Presente & & Presente & Presente & Presente & Presente \\
\hline Camachuelo & Pyrrinla pyrhula & Presente & Presente & Presente & Presente & Presente & Presente & Presente \\
\hline Reyezuelo listado & Regulus ignicapillus & - & Presente & & Abundante & & Presente & Presente \\
\hline Reyezuelo sencillo & Regulus regulus & - & Presente & Presente & - & Presente & Presente & Presente \\
\hline Chocha o Becada & Scolopax rusticola & Presente & - & & - & & & \\
\hline Verderón serrano & Serinus citrinclla & - & - & & - & & & \\
\hline Trepador azul & Sittr ewropaed & Presente & Presente & Presente & Frecuente & Presente & Presente & Presente \\
\hline Cárabo & Strix aluco & Presente & Presente & & Presente & & & \\
\hline Cumuca capirotada & Sylvia atricapilla & - & Presente & & Presente & Presente & Presente & Presente \\
\hline Curruca mosquitera & Sylvin borin & - & Presente & & Presente & Presente & Presente & Presente \\
\hline \begin{tabular}{|l} 
Urogallo \\
\end{tabular} & Tetrao torogallus & - & Presente & Presente & $\cdot$ & \begin{tabular}{|l|} 
Presente \\
\end{tabular} & Presente & \\
\hline \begin{tabular}{|l|} 
Chochín \\
\end{tabular} & Troglodyes troglodites & Presente & Presente & & Abundante & \begin{tabular}{|l|} 
Presente \\
\end{tabular} & Presente & Presente \\
\hline Zorzal alirrojo & Turdus iliacus & & & & & \begin{tabular}{|l|} 
Presente \\
\end{tabular} & & Presente \\
\hline Mirlo & Turdus merula & - & Presente & & Abundante & Presente & Presente & Presente \\
\hline Zorzal común & Turdus philomelos & Presente & Presente & & Frecuente & Presente & Presente & Presente \\
\hline Zorzal charlo & Turdus viscivorus & - & - & & Presente & Presente & PJesente & Presente \\
\hline
\end{tabular}




\begin{tabular}{|c|c|c|c|c|c|}
\hline ESPECIES & & $\begin{array}{c}\text { Nidificante } \\
\text { Hayedo } \\
\text { (Pelayo) }\end{array}$ & $\begin{array}{c}\text { Nidificante } \\
\text { Hayedo } \\
\text { (Camprodon) }\end{array}$ & $\begin{array}{l}\text { Invernante } \\
\text { Hayedo } \\
\text { (Delgado) } \\
\end{array}$ & $\begin{array}{c}\text { Fauna } \\
\text { Hayedo } \\
\text { (Jomadas) }\end{array}$ \\
\hline Nombre común & Nombre científico & Moncayo & Moncayo & Moncayo & Moncayo \\
\hline Azor & Accipiter gentilis & Escasa & - & - & - \\
\hline Gavilán & Accipiter nisus & Escasa & - & Presente & - \\
\hline Mito & Aegithalos caudatus & Frecuente & Frecuente & Presente & Presente \\
\hline Bisbita arbóreo & Anthus trivialis & & & & \\
\hline Búho chico & Asio otus & & & & \\
\hline Aguila ratonera & Buteo buteo & Muy escasa & - & - & Presente \\
\hline Chotacabras gris & Caprimulgus europaeus & Frecuente & - & - & - \\
\hline Jilguero & Carduelis carduelis & & & & \\
\hline Lúgano & Carduelis spinus & - & - & - & - \\
\hline Agateador común & Certhia brachydactya & Frecuente & Frecuente & Presente & Presente \\
\hline Agateador norteño & Certhia familiaris & - & - & - & - \\
\hline Aguila culebrera & Circaetus gallicus & Escasa & - & - & - \\
\hline Picogordo & Coccothraustes coccothraustes & & & & \\
\hline Paloma zurita & Columba oenas & - & - & - & - \\
\hline Paloma torcaz & Columba palunbus & - & Escasa & Presente & - \\
\hline Cuervo & Corvus corax & - & - & Presente & - \\
\hline Corneja & Cormus corone & - & - & $\therefore$ & - \\
\hline Cuco & Cuculus canorus & - & - & $\dot{-}$ & - \\
\hline Pico dorsiblanco & Dendrocopos leucotos & - & - & - & - \\
\hline Pico picapinos & Dendrocopos major & Frecuente & Escasa & Presente & Presente \\
\hline Pito negro & Dryocopus martius & - & - & - & - \\
\hline Escribano montesino & Emberiza cia & - & - & Presente & $=$ \\
\hline Escribano cerillo & Emberiza citrinella & - & - & - & - \\
\hline Petirrojo & Erithacus rubecula & Abundante & Abundante & Presente & Presente \\
\hline Cemícalo común & Falco tinunnunculus & - & - & Presente & - \\
\hline Papamoscas cerrojillo & Ficedula hypoleuca & - & - & - & - \\
\hline Pinzón & Fringilla coelebs & Frecuente & Abundante & Presente & Presente \\
\hline Arrendajo & Garrulus glandarius & Frecuente & Escasa & Presente & + \\
\hline Aguila calzada & Hieraius penatus & Escasa & - & - & - \\
\hline Alcaudón dorsirrojo & Lanius collurio & & & & \\
\hline Milano real & Milvus milvus & - & - & - & - \\
\hline Papamoscas gris & Muscicapa striata & - & - & - & - \\
\hline Carbonero garrapinos & Parus ater & - & Abundante & Presente & Presente \\
\hline Henerillo & Parus caeruleus & Frecuente & Abundante & Presente & $\cdot$ \\
\hline Herrerillo capuchino & Parus cristatus & - & - & - & - \\
\hline Carbonero común & Parus major & Abundante & Abundante & Presente & Presente \\
\hline Carbonero palustre & Parus palustris & - & - & - & - \\
\hline HalcÚn abejero & Pemis apivorus & Muy escasa & - & - & - \\
\hline Mosquitero papialbo & Phylloscopus bonelli & & & & \\
\hline Moquitero común & Phylloscopus collybita & Escasa & Escasa & - & Presente \\
\hline Pito teal & Picus viridis & Escasa & Escasa & Presente & - \\
\hline Acentor & Prunello modularis &.- & - & Presente & - \\
\hline Camachuelo & Pyrrlula pyrrhula & - & - & - & - \\
\hline Reyezuelo listado & Regulus ignicapillus & - & Abundante & Presente & - \\
\hline Reyezuelo sencillo & Regulus regulus & - & - & Presente & - \\
\hline Chocha o Becada & Scolopax rusticola & Muy escasa & - & - & Presente \\
\hline Verderón sertano & Serinus citrinella & - & - & Presente & Presente \\
\hline Trepador azul & Sitta europaea & Frecuente & Abundante & Presente & Presente \\
\hline \begin{tabular}{|l|} 
Cárabo \\
\end{tabular} & Strix aluco & Muy escasa & - & - & - \\
\hline Curruca capirotada & Sylvia atricapilla & Frecuente & Escasa & - & $=$ \\
\hline Curnuca mosquitera & Sylvia borin & Frecuente & - & - & - \\
\hline Urogallo & Tetrao urogallus & - & - & - & - \\
\hline Chochín & Troglodytes troglodites & Frecuente & Frecuente & Presente & - \\
\hline Zorzal alirrojo & Turdus iliacus & & & & \\
\hline Mirlo & Turdus merula & Frecuente & Frecuente & Presente & Presente \\
\hline Zorzal común & \begin{tabular}{|l} 
Turdus philomelos \\
\end{tabular} & Escasa & - & - & Presente \\
\hline Zorzal charlo & Turdus viscivorus & - & - & Presente & - \\
\hline
\end{tabular}


Tabla 2.- Otras especies detectadas en hayedos eurosiberianos y en el hayedo del Moncayo durante las Jornadas

\begin{tabular}{|c|c|c|c|c|c|c|}
\hline \multicolumn{2}{|l|}{ ESPECIES } & $\begin{array}{c}\text { Fauna } \\
\text { Hayedos } \\
\text { (Gómez) }\end{array}$ & $\begin{array}{c}\text { Fauna } \\
\text { Hayedos } \\
\text { (Balcells) }\end{array}$ & $\begin{array}{c}\text { Fauna } \\
\text { hayedo } \\
\text { (Jornadas) }\end{array}$ & & \\
\hline Nombre común & Nombre científico & P. Ibérica & P.N. Ordesa & Moncayo & Corología & Exigencias \\
\hline Armiño & Mustela erminea & - & Presente & - & Eurasia & $\begin{array}{l}\text { Bosques y zonas descubiertas } \\
\text { cercanas }\end{array}$ \\
\hline Corzo & Capreolus capreolus & Presente & - & Presente & Paleártico Cosmopolita & Forestal denso y húmedo \\
\hline Culebra lisa Europea & Coronella austriaca & & Presente & & Paleártico & Cosmopolita. Claros, roquedos \\
\hline Garduña & Martes foind & Presente & & Presente & Holoártico & $\begin{array}{c}\text { Bosques frondosas pero orilla } \\
\text { y pedreras }\end{array}$ \\
\hline Garduña of foina & Martes martes & - & Presente & - & Eurasia & $\begin{array}{c}\text { Grandes bosques, prefiere } \\
\text { coníferas }\end{array}$ \\
\hline Lagartija roquera & Lacerta muralis & & Presente & & & Cosmopolita \\
\hline \begin{tabular}{|l} 
Lagartija serrana \\
\end{tabular} & Lacerta monticola & & & Presente & Centroeuropeo & Linderos bosques \\
\hline Lagarto verde & Lacerta viridis & & Presente & & & \\
\hline Lirón gris & Gtis glis & - & Presente & - & Holoártico & $\begin{array}{c}\text { Bosques caducifolios buen } \\
\text { sotobosque }\end{array}$ \\
\hline Lucién & Anguis fragilis & & - & Presente & & - \\
\hline \begin{tabular}{|l|} 
Musaraña \\
colicuadrada \\
\end{tabular} & Sorex araneus & - & Presente & - & & Herbazales \\
\hline Musaraña enana & \begin{tabular}{|l} 
Sorex minutus \\
\end{tabular} & & Presente & - & Europeo & Herbazales \\
\hline Musgaño patiblanco & Neomys fodiens & - & Presente & - & Centroeuropeo & $\begin{array}{l}\text { Cauces altos y medios de } \\
\text { montaña }\end{array}$ \\
\hline Ratón de campo & Apodemus sylvaticus & - & Presente & - & Holoártico, montano & - \\
\hline Ratón leonado & Apodemus flavicolis & - & Presente & - & Holoártico Cosmopolita & Forestal \\
\hline Vibora & Vipera aspis & & Presente & Presente & Europeo & Zonas abiertas y pedregosas \\
\hline
\end{tabular}

Se hacía imprescindible para el estudio que nos hemos planteado, contar con información previa sobre el enclave sometido a estudio y sobre otros enclaves en los que se encontrasen masas de hayedos puros, que permitieran contrastar la información generada a lo largo de las "Jornadas" y en trabajos anteriores (ver bibliografía).

La mayoría de los trabajos consultados tanto para el Moncayo, como para otras masas de hayedo, hacen referencia a la ornitofauna, de ahí que nuestro trabajo se centre en este grupo faunístico, sin olvidar como referencia los datos de otros grupos. Por un lado, para recopilar datos acerca de la ornitofauna presente en el hayedo, se realizaron transectos o taxiados (TELLERÍA, 1977) en su interior, aprovechando la existencia de una pista forestal, poco utilizada, que lo recorre en varias direcciones. Se anotaban todos los contactos visuales y auditivos observados a ambos lados de la pista, en una banda de $25 \mathrm{~m}$ a cada lado del itinerario. Estos recorridos se realizaron a primera hora de la mañana, coincidiendo con la mayor actividad de las aves y unas veces se hizo a pie y otras en vehículo, llevando a cabo paradas para realizar escuchas, durante 10 minutos, cada 200 metros. 
Tabla 3.- Aspectos corológicos y ecológicos de la avifauna presente en hayedos

\begin{tabular}{|c|c|c|c|c|}
\hline Nombre científico & Nombre común & Corologia & Alimentación & Hábitat \\
\hline Accipuer gentilis & Azor & Holoártico & Aves, mamíferos, reptiles & Forestal, extenso \\
\hline Accipiter nisus & Gavilán & Paleártico & Aves, mamíferos, anfibios & Forestal, menos extenso \\
\hline Aegithalos catudatus & Mito & Paleârtico & Artrópodos, semillas & Forestal con sotobosques importante \\
\hline Anthus trivialis & Bisbita arbóreo & Europeo-turquestani & Artrópodos & Borde bosques \\
\hline Asio otus & Búlıo chico & Holoártico & Micromamíferos & Bosques claros y sotos \\
\hline Buteo buteo & Ratonero & Holoártico & Mamiferos & Forestal no exclusivo \\
\hline Caprimulgus europaeus & Chotacabras gris & Paleârtico & Insectivoro & Boscues con claros \\
\hline Carduelis spinus & Lúgano & Paleártico & Granívoro & Bosques mixtos \\
\hline Certhia brachydactya & Agateador común & Holoártice & Insectívoro & Forestal, coníferas \\
\hline Certhia familiaris & Agateador norteño & Holoártico & Insectívoro & Forestal, caducifolios \\
\hline Circaetus gallicus & Aguila culebrera & Indo-africano & Ofidios & Cosmopolita, campo abierto \\
\hline Columba oenas & Paloma zurita & Europeo-turquestanl & Frugívora & Forestal \\
\hline Columba palumbus & Paloma torcaz & Europeo-turquestanl & Frugívora & Cosmopolita, forestal \\
\hline Corvus corax & \begin{tabular}{|l} 
Cuervo \\
\end{tabular} & Holoártico & & Cosmopolita \\
\hline Corvus corone & Comeja & Paleártico & & Áreas cultivadas \\
\hline Cuculus canorus & Cuco & Paleartico & Orugas & Cosmopolita \\
\hline Dendrocopos lencotos & Pico dorsiblanco & Paleártico & Insectos xilófagos & Bosques caducifolios extensos y maduros \\
\hline Dendrocopos major & Pico picapinos & Paleártico & Xilófagos, frutos secos & Bosques extensos y maduros, frec hayedos \\
\hline Dryocopus martius & Pito negro & Paleártico & larvas insectos xilófagos & Bosques caducifolios extensos y maduros \\
\hline Emberiza cia & Escribano montesino & Paleártico & & Áreas despejadas \\
\hline Enberiza citrinella & Escribano cerillo & Paleártico & & Matorrales norteños \\
\hline Erithacus rubecuia & Petirrojo & Centroeuropeo & & Bosque y matorral \\
\hline Falco inumnunculus & Cemícalo común & Paleártico & & Cosmopolita no forestal \\
\hline Ficedula hypoleuca & Papamoscas cerrojillo & Centroeuropeo & & Claros de bosques maduros \\
\hline Fringilla coelebs & Pinzón & Centroeuropeo & & Cosmopolita forestal \\
\hline Garrulus glandarius & Arrendajo & Paleártico & & Cosmopolita forestal \\
\hline Hieratus permatus & Aguila calzada & Turquestano-medit. & Oruitófaga & Bosques inontanos \\
\hline Lanias collurio & Alcandón dorsirrojo & Paleártico & & Matorral de enclaves húmedos \\
\hline Milvus milvus & Milano real & Europeo & & Cosmopolita \\
\hline Muscicapa striata & Papamoscas gris & Europeo-turquestani & & Bosques \\
\hline Pailus ater & Carbonero garrapinos & Paleártico & & Bosques de coníferas \\
\hline Parus caeruleus & Herrerilio & Europeo & & Bosques húmedos, sotos, jardines \\
\hline Parus cristatus & Herrerillo capuchino & Europeo & & Cosmopolita forestal \\
\hline Parus major & Carbonero común & Paleártico & & Cosmopolita forestal \\
\hline Parus palustris & Carbonero palustre & Paleártico & & Bosques montanos húmedos \\
\hline Pernis apivorus & Halcón abejero & Europeo & & Bosques montanos húmedos \\
\hline Phylloscopus collybita & Moquitero común & Paleártico & & Bosques montanos húmedos \\
\hline Picus viridis & Pito real & Europeo & & Cosmopolita forestal \\
\hline Prunella modularis & Acentor & Europeo & & Bosques y matorrales \\
\hline Pyrrhula pyrhula & Camachuelo & Paleártico & & Boques montanos húmedos, hayedos \\
\hline Regulus ignicapillus & Reyezuelo listado & Holoártico & Artrópodos & Boques montanos húmedos, hayedos \\
\hline Regulus regulus & Reyezuelo sencillo & Paleártico & Aitrópodos & Bosques montanos húmedos, coníferas \\
\hline Scolopax rusticola & Chocha o Becada & Paleártico & Lombrices & Bosq. húmedos, hayas, roble, suelo fresco \\
\hline Serinus citrintella & Verderón serrano & & & \\
\hline Sitta europaea & Trepador azul & Paleártico & Insectivoro cortezas & Bosques frondosos maduros \\
\hline Stix aluco & Cárabo & Paleártico & Micromamíferos & Forestal \\
\hline Sylvia atricapilla & Curruca capirotada & Europeo & & Sotobosque denso \\
\hline Sylvia borin & Curruca mosquitera & Europeo & & Sotobosque denso \\
\hline Tetrao urogallus & Urogallo & Paleártico & & Bosques tranquilos con abundantes bayas \\
\hline Troglodytes troglodites & Chochín & Holoártico & & Forestal sotobosque denso \\
\hline Turdus merula & Mirlo & Paleártico & & Cosmopolita, sotobosque, sotos \\
\hline Turdus philomelos & Zorzal común & Europeo & & Forestal sotobosque \\
\hline Turdas viscivorus & Zorzal charlo & Europeo-turquestaní & & Folestal, bordes y claros \\
\hline
\end{tabular}


Por otro lado, se anotó toda la información en forma de huellas y deyecciones encontradas en nuestros transectos y en las incursiones realizadas por el interior del hayedo, completandó esto último, mediante visitas a horas nocturnas, lo cual nos proporcionó una importante información acerca de la presencia de determinados mamíferos. Toda la información generada se ha completado con trabajos realizados con anterioridad en la zona, con la finalidad de cubrir el espectro faunístico presente en el hayedo. Posteriormente se ha contrastado con la información extraida de trabajos elaborados en otros hayedos, localizados en zonas no limítrofes como la que nos ocupa, lo cual nos permitió constatar la presencia o ausencia de determinadas especies, que por su ecología, actúan como bioindicadores de la situación biogeográfica en la que se encuentra el hayedo del Moncayo.

No se trata de establecer una comparativa de tipo cuantitativa, sino cualitativa, para lo cual se elaboró una tabla de doble entrada, en la que se situaron como registros las diferentes especies detectadas durante nuestro trabajo y presentes en los trabajos consultados para el hayedo del Moncayo, junto a las de otros más eurosiberianos, mientras que como campos se situaron los diferentes hayedos consultados (Tabla 1). Esta tabla permitió de forma rápida detectar las especies ausentes en el hayedo del Moncayo, pero presentes en hayedos más eurosiberianos. La comparativa se llevo a cabo tan sólo con la ornitofauna, debido a que tan sólo de ésta se disponía la información más completa, quedando el resto de datos recogidos en una tabla aparte (Tabla 2).

\section{DISCUSIÓN}

Para la comarca del Moncayo se han descrito un total de 174 especies, repartidas de la siguiente forma: 27 anfibios y reptiles, 120 aves nidificantes y 27 especies de mamíferos y quirópteros (CAMPODRON, 1995).

En nuestro trabajo y por las razones ya explicadas en el apartado de metodología, tendremos en cuenta la avifauna presente en la comarca, que supone el $68 \%$ de las especies citadas, así como su caracterización biogeográfica (Gráfico 1).

El reparto que se observa en el gráfico de distribución de los tipos corológicos de la fauna de la comarca del Moncayo (Gráfico 1), revela la situación transicional de la zona. Como puede observarse, los diferentes ambientes se encuentran muy cercanos en el porcentaje del total de especies, quedando las eurosiberianas ligeramente por debajo del resto $(22 \%)$, no llegando a suponer una tercera parte del total, corrobora la situación anteriormente descrita de la importante presencia de especies eurosiberianas en el Macizo del Moncayo. 


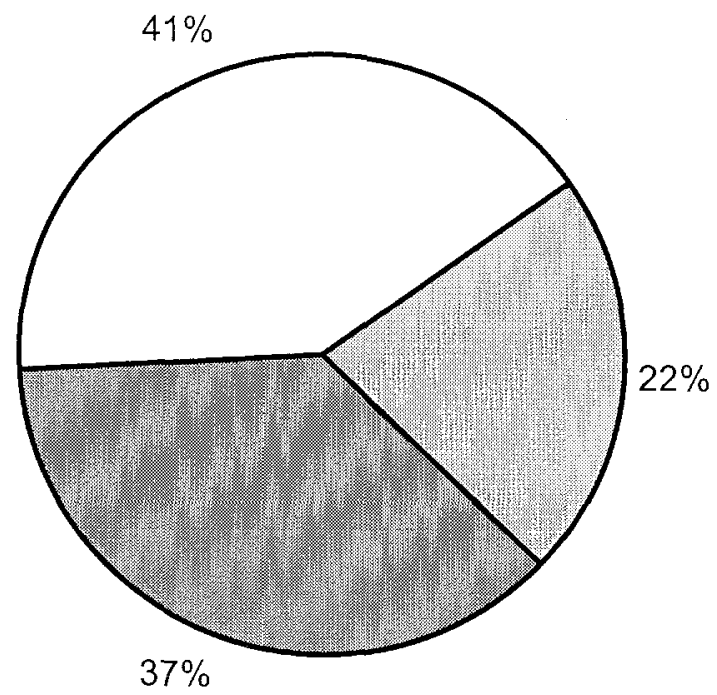

Gráfico 1.- Tipos corológicos de la avifauna de la comarca del Moncayo. Fuente: CAMPODRÓN, 1998.

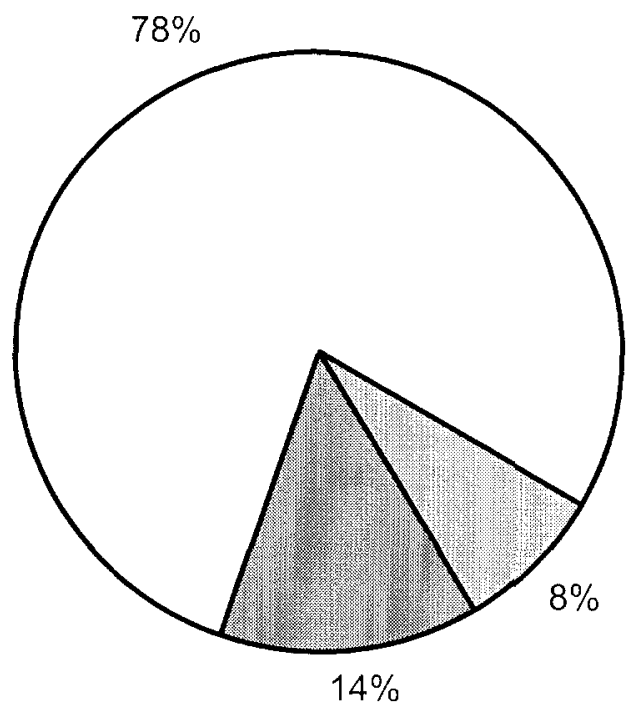

Gráfico 2.- Distribución de los tipos corológicos de la avifauna detectada en el hayedo del Moncayo. Fuente: CAMPODRÓN, 1998. 


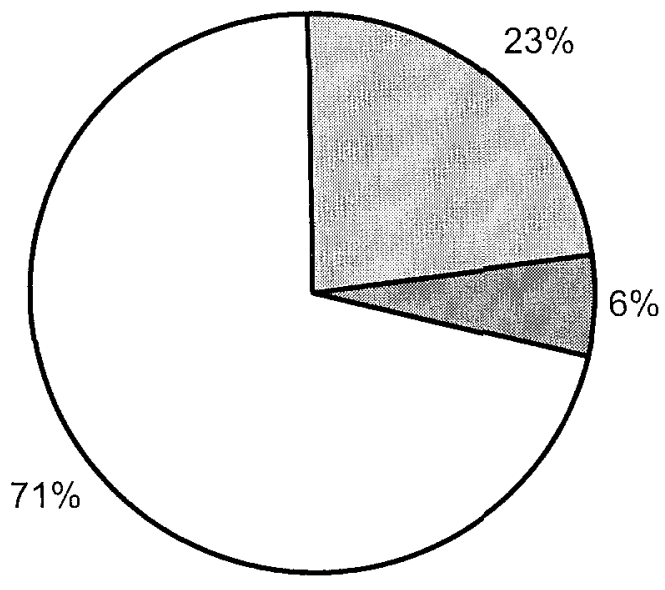

Gráfico 3.- Distribución de la avifauna de los hayedos pirenaicos.

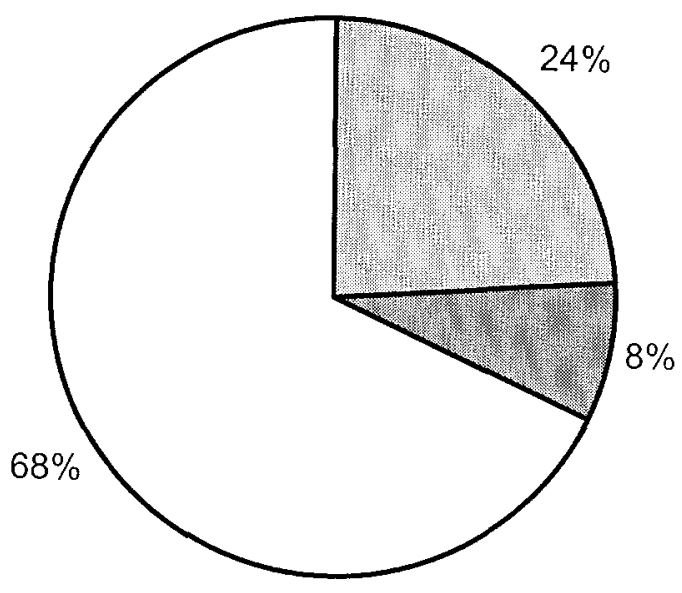

Gráfico 4.- Distribución de la avifauna de los hayedos cantábricos. 
Si relacionamos la ornitofauna citada para la comarca del Moncayo con la presente en el hayedo sometido a estudio (Gráficos 1 y 2), se observa una importante disminución en el hayedo de las especies denominadas eurosiberianas $(8 \%)$ respecto al total de la comarca (22\%), adquiriendo una mayor importancia en el hayedo el espectro cosmopolita $(78 \%)$, que aumenta considerablemente respecto a la situación en la comarca $(41 \%)$, mientras que el tipo mediterráneo se reduce más del doble (del $37 \%$ al $14 \%)$.

Esta misma situación de aumento de las especies cosmopolitas, se repite en las distribuciones para hayedos pirenaicos y cantábricos (Gráficos 3 y 4), aunque en estos hay que reseñar que se produce un aumento del porcentaje de las especies eurosiberianas ( $23 \%$ y $24 \%$ ), del mismo modo que una mayor reducción del espectro mediterráneo en ambos casos.

La mayor presencia de aves del grupo denominado cosmopolita en los hayedos, se debe a que estas especies son capaces de aprovechar un amplio abanico de ecosistemas, no siendo la formación vegetal que nos ocupa una excepción, sobre todo en ambientes como en el macizo del Moncayo, donde convive una amplia variedad de ecosistemas, en su mayoría forestales, que presentan una menor dificultad de colonización por ser sus exigencias ecológicas menos duras que los medios abiertos de condiciones más extremas. Del mismo modo, al contrario que ocurre con otros medios forestales, los hayedos en invierno son seleccionados por encima de otro tipo de bosques por un mayor número de fauna, debido a una mayor disponibilidad de recursos tróficos (ÁLVAREZ, 1993), aumentando de forma notable el número de aves esencialmente cosmopolitas, como zorzales y fringílidos.

Por otra parte, el espectro mediterráneo sufre un importante descenso en el hayedo del Moncayo respecto a la comarca (Gráficos 1 y 2), descenso que es mucho más acusado en los hayedos pirenaicos y cantábricos (Gráficos 3 y 4). Las especies mediterráneas y submediterráneas lo tienen difícil para colonizar ambientes eurosiberianos, la climatología que favorece su existencia y la composición y estructura de la comunidad vegetal, no les favorece, aun así en el hayedo del Moncayo, al tratarse de un medio forestal poco extenso y rodeado de condiciones y hábitats mediterráneos, no es extraño una presencia importante de este grupo biogeográfico, que lo utiliza de forma puntual, como refugio o en busca de recursos tróficos, hecho este que no se repite en los hayedos pirenaicos y cantábricos, donde podemos describir esta presencia como testimonial, ya que sus porcentajes no llegan a representar un $10 \%$ del total en ningún caso.

Por último, el grupo denominado eurosiberiano muestra variaciones significativas en cuanto a los diferentes espacios considerados. Se observa una importante presencia de éste en toda la comarca, con un $22 \%$, mucho mayor de lo que 
se extrae del gráfico del hayedo del Moncayo (8\%). Este dato no debe de extrañar, pues en el gráfico de la comarca también aparecen reflejadas las especies presentes en los pinares subalpinos, acebales, enebrales, piornales y prados de cumbre presentes en el macizo del Moncayo, que introducen nuevas especies eurosiberianas al listado de aves de la comarca y que por sus características ecológicas no explotan el hayedo, buscando zonas de pinar y espacios abiertos.

Sin embargo, llama la atención la importante diferencia que existe entre el porcentaje eurosiberiano del Moncayo (8\%) y los pirenaicos $(23 \%)$ y cantábricos $(24 \%)$, que ponen de manifiesto la diferencia en la avifauna del hayedo "límite o relicto" del Moncayo y los situados en territorios eminentemente eurosiberianos.

Este importante contraste da paso al principal objetivo de nuestro trabajo, establecer el porque de esta situación, para lo cual listamos las especies citadas en los diferentes enclaves, atendiendo a su corología y hábitat (Tabla 3). Este listado permitirá establecer las exigencias de las aves de cada enclave y descubrir las ausencias más relevantes en el Moncayo.

A pesar de observarse un porcentaje extremadamente bajo de especies eurosiberianas, en la composición ornítica del hayedo del Moncayo se encuentran especies claramente ligadas a este tipo de formación boscosa: la chocha perdiz, becada o sorda (Scolopax rusticola) (Mapa 2), que necesita bosques húmedos, diversificados y con suelos profundos, frescos y sueltos, en el que el humus sea abundante y permita el desarrollo y la búsqueda de los invertebrados que conforman su dieta (CARDELUS, 1997), el halcón abejero (Pernis apivorus) que busca formaciones de frondosas, con presencia de claros y que es muy sensible a la alteración de su hábitat (Mapa 3), o el trepador azul (Sitta europaea), insectívoro y frugívoro, presente en diversos tipos de bosque, aunque en los hayedos encuentra las mejores poblaciones (CARDELUS, 1997).

La presencia de las especies citadas en el párrafo anterior confirma el carácter eurosiberiano de la formación boscosa, aunque del total de la avifauna listada (Tabla 1), hay que destacar la ausencia en el Moncayo de ocho especies de distribución eurosiberiana, presentes en el resto de hayedos más atlánticos: agateador norteño (Certhia familiaris), pico dorsiblanco (Dendrocopos leucotos), pito negro (Dryocopus martius), papamoscas cerrojillo (Ficedula hypoleuca), carbonero palustre (Parus palustris), camachuelo común (Pyrrhula pyrrhula), urogallo (Tetrao urogallus) y zorzal alirrojo (Turdus iliacus), de todas ellas no tendremos en cuenta al urogallo y el pico dorsiblanco (Mapa 4), debido a su distribución puntual y situación relicta en la Península lbérica. 


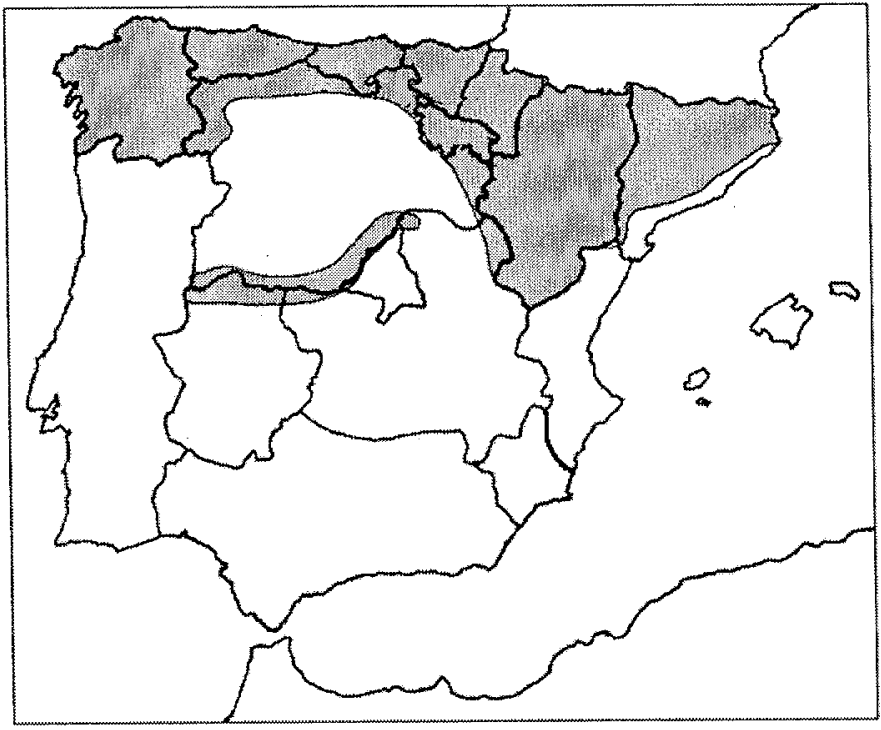

Mapa 2. Distribución de Chocha perdiz (Scolopax rusticola). Fuente: CARDELUS, 1997.

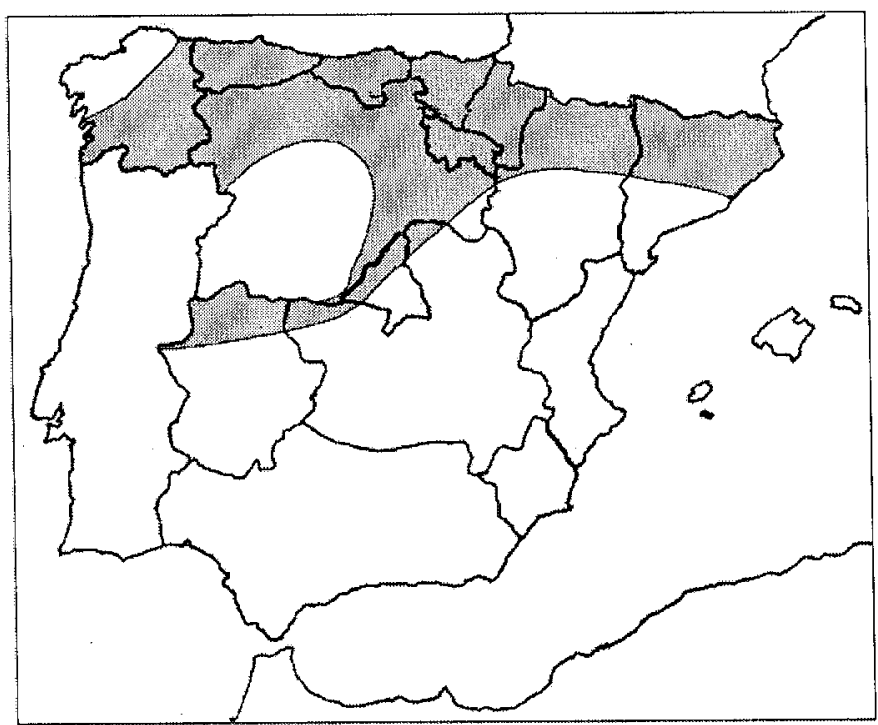

Mapa 3. Distribución de Halcón abejero (Pernis apivorus). Fuente: CARDELUS, 1997. 


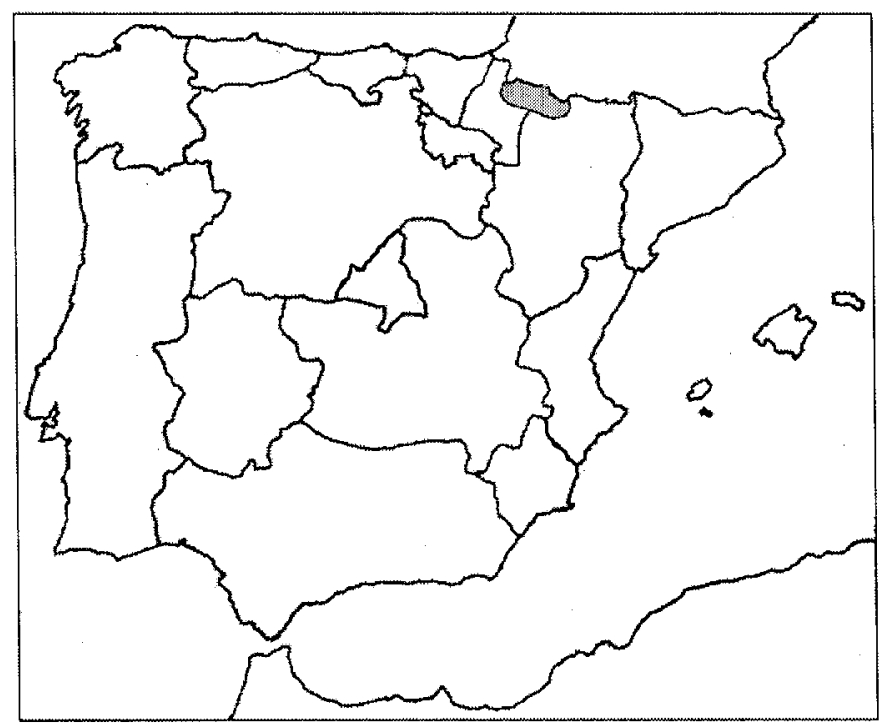

\section{Mapa 4. Distribución de Pico dorsiblanco (Dendrocopos leucotos). Fuente: CARDELUS, 1997.}

Descartadas las dos aves, resultan significativas las ausencias de las otras seis especies citadas anteriormente, ya que se trata de aves con exigencias ecológicas muy concretas, por lo que servirán como bioindicadores acerca de la situación ecológica de la zona de estudio.

El análisis de las características ecológicas de estas especies (Tabla 3), muestra unas preferencias muy concretas que nos aproximan a comprender su ausencia. Todas ellas necesitan de bosques caducifolios o montano húmedos para desarrollarse, con la excepción del zorzal alirrojo, que sólamente se aprovecha de estos ecosistemas en época invernal, en busca de recursos tróficos consistentes en frutos y bayas, abundantes en este tipo de formaciones boscosas. El resto necesitan hábitats bien desarrollados, hayedos bien estructurados, formaciones maduras, extensas, con un sotobosque denso que ofrezca cobijo y alimento, diversificando el espectro aviar.

Ejemplo de ello son las ausencias de agateador norteño, pito negro y papamoscas cerrojillo, que necesitan de bosques maduros, en los que coexista madera muerta que les provea de alimento, así como ejemplares de árboles de gran porte con hoquedades que favorezcan su nidificación. Podría pensarse que estas ausencias están causadas por la ubicación aislada, o de límite de distribución del macizo del Moncayo, pero la cercanía en la Cordillera Ibérica de enclaves donde se citan estas especies, permite descartar esta hipótesis. 


\section{CONCLUSIONES}

Como apunta DIAZ (1997), las tendencias de las aves forestales de la Península parecen relacionarse con algunos rasgos de nuestros bosques, climáticos o antrópicos (edad de la masa forestal, densidad de árboles y matorrales, extensión). Los pajaros carpinteros presentan patrones de distribución en la Península Ibérica en los que destacan la disminución de especies y abundancias en sentido norte-sur, esto se explica por requerimientos de hábitat (ausencia o escasa extensión de los hayedos para los picos dorsiblancos), disminución de recursos o aumento de predadores, así como incapacidad de colonizar lugares adecuados por lo alejado de los núcleos estables.

El análisis comparativo entre la aves presentes en el hayedo del Moncayo y las de otros hayedos puros nos revela la ausencia de determinadas especies, que por su ecología aportan información acerca del estado de este bosque. No es coincidencia que no se localicen aquellas que necesitan de masas forestales maduras y denso sotobosque, donde encuentran con facilidad huecos para nidificar y abundantes recursos tróficos generados por la existencia de una formación boscosa bien estructurada y con presencia de arbolado viejo, pues el hayedo sometido ha estudio es un bosque joven, talado hasta épocas recientes, testigo de ello son las frecuentes huellas de carbóneo que se pueden encontrar en su interior, así como la presencia de abundantes ejemplares con varios pies y la ausencia de ejemplares viejos de gran porte.

Esto coincide con la situación descrita por PURROY (1977), el cual establece una densidad media para los hayedos pirenáicos de 68 aves/10 Has, observando una mayor diversidad de aves en el Pirineo occidental (66'2 aves/10 Has en Olaldea, 81, 6 aves/10 Has en Leyre), en los hayedos más puros, mientras que el central, más pobre, con una importante ausencia de arbolado con huecos y un estrato arbustivo menos importante, presenta una densidad de aves más baja (16'8 aves/10 Has en Ordesa, 24 aves/ 10 Has en Artiga de Lin).

Para el hayedo del Moncayo CAMPODRON et al. (1989) establece como ave más abundante al petirrojo, muy por encima del resto de aves que le siguen: reyezuelo listado, pinzón, carbonero común, trepador azul, herrerillo común y carbonero garrapinos, siendo esta formación arbórea la menos poblada de la comarca, con densidades más bajas (37,84 aves / 10 Has.).

DELGADO (1989), señala que los hayedos presentan una abundancia y diversidad de aves indicadora de la disponibilidad de recursos alimenticios suficientes y diversos. No es de estrañar por tanto, que no estén presentes en el Moncayo especies eurosiberianas tan selectivas en su hábitat como: agateador norteño, pito negro y papamoscas cerrojillo, que necesitan de bosques maduros con presencia de hoquedades para nidificar y árboles muertos que faciliten la presencia de larvas, insectos xilófagos y 
hormigas, principales componentes de su dieta, o como el Carbonero palustre, con una distribución en la Península ligada a los bosques caducifolios, maduros y densos, presente en el Sistema Ibérico tan sólo en los rodales maduros de hayas (DE JUANA, 1980).

Todo ello nos hace pensar que situación corológica, relicta o de límite de distribución del hayedo del Moncayo, no es la principal causa de la menor presencia de una fauna claramente eurosiberiana, sino que corresponde a aspectos climácicos, de madurez de la formación, siendo esta la principal razón detectada, que impide la presencia de los grandes especialistas eurosiberianos. Es la rarefacción de las formaciones maduras lo que provoca la desaparición, desplazamiento y disminución de las áreas de influencia de la fauna más especializada.

\section{BIBLIOGRAFÍA}

ÁlVAREZ, A. y PURROY, F. J.: "Comparación de las comunidades de aves nidificantes e invernantes en los medios forestales de la Cordillera Cantábrica-leonesa". Ecología, 7. ICONA.

BALCELLS, E. (1993): Las hayas y los hayedo-abetares en el entomo del parque nacional de Ordesa y Monte Perdido. Instituto Pirenaico de Ecología. Jaca.

BURGAZ, A., FUERTES, E., y MENDIOLA, A. (1985): "Esquema de la gradación altitudinal de la vegetación del Macizo del Moncayo (Zaragoza-España)". Studia Botanica IV.

CAMPODRON, J., MORENO, J. y OMEÑACA, A. (1989): "Distribución ecologica y corología de las aves nidificantes del Moncayo". Turiaso IX.

CARDELÚS, B. (dir.) (1997): Los Bosques del Norte en La España Salvaje. vol. 3. Edit. Planeta.

COSTA, L. (1997): Evolución estacional de la avifauna en hayedos de la montaña cantábrica. Departamento de Biología Animal. Universidad de León.

DELGADO, I. (1989): "Comunidad de aves invernantes en un hayedo del Moncayo". Turiaso IX.

GÓMEZ, F. (coord.) (1997): Los bosques ibéricos. Ed. Planeta.

MONTSERRAT, P. (1984): "Flora". Enciclopedia Temática de Aragón, tomo 6. Edit. Moncayo. Zaragoza

PEDROCCHI, C. (1978): Las aves de Aragón. Librería General. Colección Aragón. 
PEDROCCHI, C. (1987): Fauna ornitica del Alto Aragón Occidental. Monografías del Instituto Pirenaico de Ecología, 1. C.S.I.C. Jaca.

PELAYO, E.: Aves nidificantes de las comarcas de Borja y Tarazona: hábitat, distribución y estado de conservación. Diputación General de Aragón.

PELliCER, F. (1988): El Moncayo. Colección Mariano de Pano y Ruata. Caja de Ahorros de la Inmaculada. Zaragoza.

PURROY, F.J. (1977): "Avifauna nidificante en hayedos, quejigales y encinares del Pirineo". Boletín de la Estación Central de Ecologia, 11.

PURROY, F.J. et al. (1990): "Bosque y fauna de vertebrados terrestres en España. Ecología", Fuera de serie 1.

TELLERIA, J.L. (1986): Manual para el censo de los vertebrados terrestres. Edit. Raices. Madrid. 